\title{
GOBLIT: A GIANT OPTO-MECHANICAL BUULK-MACHINED LIGHT TRANSDUCER
}

\author{
Ajay K. Bhat, Laura C. Fegely and Sunil A. Bhave \\ OxideMEMS Lab, Cornell University, Ithaca, NY, USA
}

\begin{abstract}
We report guided-light opto-mechanical sensing of the mechanical vibrations of a toroidal-lipped $\mathrm{SiO}_{2}$ GOBLiT. An optical quality factor of $1.7 \times 10^{5}$ allowed us to measure mechanical vibrations of the GOBLiT with mechanical quality factor 206 in air for the $n=2$ degenerate wineglass mode. Light was coupled into the resonator via a tapered optical fiber and used to sense mechanical motion of the GOBLiT. This result shows the viability of the toroidal-lipped GOBLiT for applications such as opto-mechanical gyroscopes.
\end{abstract}

\section{INTRODUCTION}

In recent years there has been much interest in optomechanical systems as displacement sensors, mass sensors, inertial sensors and oscillators. Shot noise limited displacement sensitivity of $10^{-19} \mathrm{~m} . \mathrm{Hz}^{-1 / 2}$ has been shown in microresonators [1]. Usually these devices have been two-dimensional disk type structures anchored at the center. Their mechanical quality factor is limited by anchor loss due to direct coupling of the anchor to the resonance modes. They also have various parasitic out-of-plane mechanical modes that populate the frequency spectrum. One solution proposed to solve these problems is to use a three dimensional wineglass structure where the anchor is decoupled from the mechanical modes of interest and the structure does not have any parasitic modes [2]. Many sensor applications require highly isotropic stiffness and damping properties [3], as well as high mechanical quality factors, ruling out conventional electrostatically transduced materials [4][5].

The performance of an optomechanical device is strongly dependent on optical quality factor. Optical losses depend on material properties, surface roughness and the curvature of the optical cavity. 2D optical disk structures such as [1] have used a $\mathrm{CO}_{2}$ laser to selectively heat the oxide and reflow the rim of the disk to form a toroid. This improves the optical quality factor by orders of magnitude. The GOBLiT presented in this paper seeks to combine the toroidal optical cavity with the 3D mechanical resonator to achieve high quality device performance.

\section{FABRICATION}

The GOBLiT fabrication process flow is shown in Fig. 1. The resonator consists of an oxide hemispherical shell with an extended lip melted into a toroid at the outermost circumference, supported at the base by a silicon pedestal as shown in the SEM in Fig. 1g). The mechanical quality factor and frequency split of degenerate modes of the $n=2$ wineglass mode will be heavily dependent on the isotropy and smoothness of the bowl. In order to achieve better mode-matching which is necessary for gyroscopes, we start with a $<111>$ silicon wafer to form the mold, as shown in [6][7]. After alignment marks are patterned, a masking layer of stoichiometric nitride is deposited using LPCVD. Once patterned, an HNA etch is used to create hemispherical molds within the bulk of the silicon wafer. Hot phosphoric acid is used to remove the nitride mask, thermal oxide is grown to a thickness of $1.1 \mu \mathrm{m}$, and the lip of the GOBLiT is patterned. The device is then partially undercut using $\mathrm{XeF}_{2}$ and heated with a $\mathrm{CO}_{2}$ laser to reflow the oxide at the edge of the lip. The $\mathrm{XeF}_{2}$ partial undercut is crucial in controlling the uniformity of the reflow. Any difference in thermal path length a)

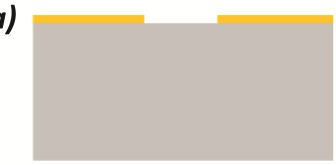

b)



c)

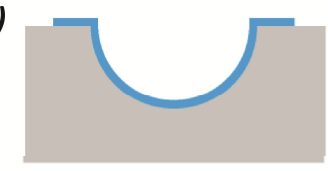

d)

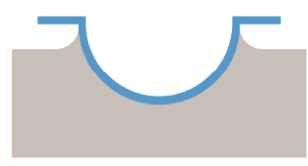

e)



f)



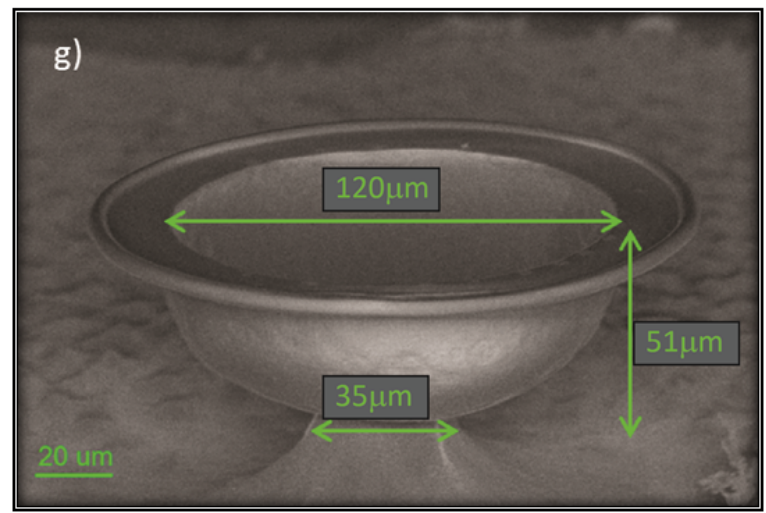

Figure 1: a) LPCVD silicon nitride mask is patterned with the etch hole. b) Silicon is isotropically etched in HNA. c) Thermal silicon dioxide is grown and the lip patterned. d) Partial undercut etch of silicon in $\mathrm{XeF}_{2}$. e) Oxide is reflown using a $\mathrm{CO}_{2}$ laser to form the toroidal optical cavity. f) Device is timed-etched in $\mathrm{XeF}_{2}$ to define the anchor. $g$ ) SEM of a final device.

will cause inconsistent reflow, so the GOBLiT is only undercut up to the edge of the bowl. Undercutting any further will greatly exaggerate any path length differences between the rim and bowl due to the $3 \mathrm{D}$ shape of the device. Once the toroidal lip has been formed, $\mathrm{XeF}_{2}$ etching is continued to release the device for measurements.

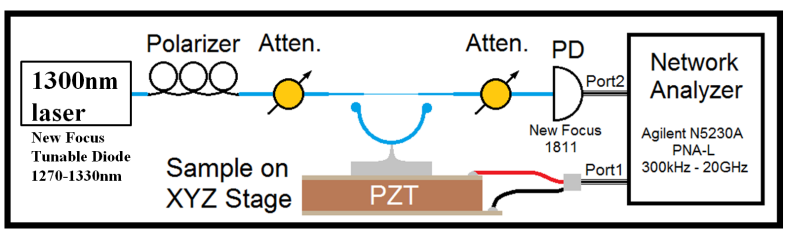

Figure 2: Schematic of the measurement setup for optically detecting the mechanical motion of the GOBLiT. Port 1 of the network analyzer is used to drive the PZT stage which in turn drives the mechanical modes of the GOBLiT. The mechanical motion intensity modulates the light in the tapered fiber which is detected by the photodetector and subsequently measured using port 2 of the network analyzer. 


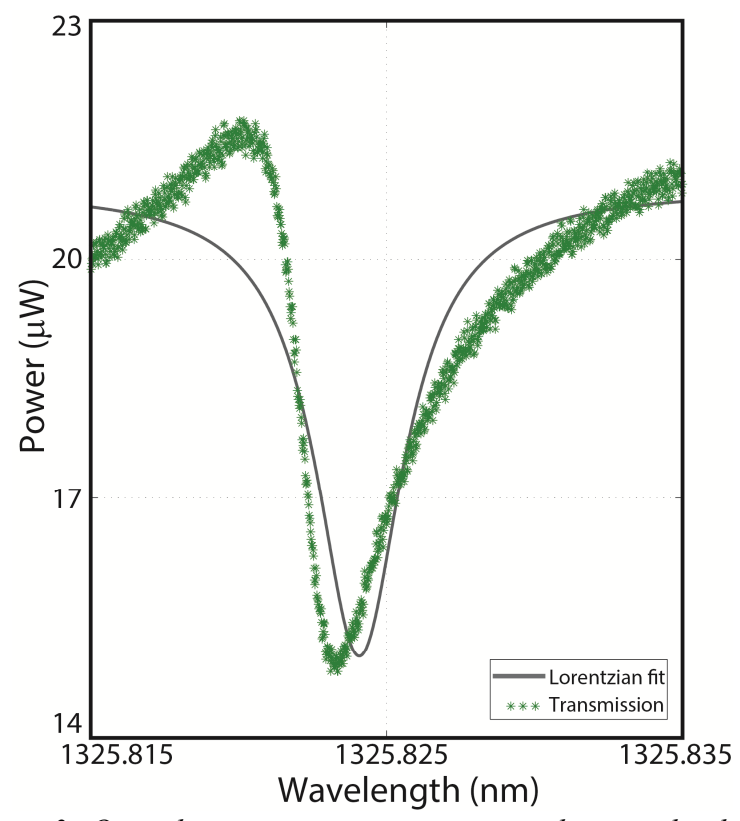

Figure 3: Optical transmission measurement showing the dip at optical resonance $(1325.823 \mathrm{~nm})$ of the cavity with an optical quality factor $1.7 * 10^{5}$.

\section{EXPERIMENT}

The toroidal lip forms the optical cavity of the GOBLiT. We use a tapered fiber to couple light into the GOBLiT, and interrogate it. Fig.2 shows a schematic of this measurement scheme. To obtain the tapered fiber we start with a $125 \mu \mathrm{m}$ diameter single mode (1550nm wavelength) optical fiber. We then use a hydrogen flame to heat a portion of the fiber and simultaneously pull from the two ends to draw out the fiber. The transmission of the $1300 \mathrm{~nm}$ laser through the fiber is continuously monitored during this process and the heating and pulling is stopped when the fiber is single mode. At this point the diameter of the fiber is $\sim 1.3 \mu \mathrm{m}$ with a glass "core" and an air "cladding". A part of the optical mode now sits outside the fiber with the amplitude falling off exponentially in the radial direction. This evanescent portion of the optical mode can now be used to couple light into the optical cavity when the GOBLiT is in the near-field of the taper.

The governing equations of motion of the optical field and the mechanical mode are [8],

$$
\begin{aligned}
& \dot{a}=i(\Delta+G x) a-\frac{\kappa}{2} a+\sqrt{\kappa_{e x}} s \\
& \ddot{x}+\Gamma_{\mathrm{m}} \dot{x}+\omega_{m}^{2} x=\frac{F_{\text {drive }}}{m}
\end{aligned}
$$

where, $a$ is the normalized electric field amplitude of the optical field inside the cavity, $\Delta$ is the detuning of the laser from the optical cavity resonance $\left(\omega_{\mathrm{L}}-\omega_{\mathrm{C}}\right), G$ (defined as $-\delta \omega_{\mathrm{c}} / \delta \boldsymbol{l}$ where $\delta \boldsymbol{l}$ is the change in cavity length) is the optomechanical coupling factor, $\kappa$ is the total optical loss rate, $\kappa_{e x}$ is the coupling rate of the optical cavity to the external environment, i.e. the tapered fiber, and $s$ is the normalized electric field amplitude of the input laser drive. The transmitted field amplitude is given by

$$
a_{\text {out }}=s-\sqrt{\kappa_{\text {ex }}} a
$$

is monitored with a photodetector. When the laser drive scans across a resonance mode of the optical cavity, we measure a transmission dip as shown in Fig. 3. The mechanical mode is modeled as a driven simple harmonic oscillator in equation (2). In our setup, the driving force comes from the piezoelectric stage of the sample. From the equations it is clear that the mechanical motion modulates the optical field amplitude and this is picked up as an intensity modulation of the detected transmission at the photodetector. This measurement is performed using a network analyzer in an $\mathrm{S}_{21}$ configuration with port 1 being the piezo drive and port 2 being the photodetector signal (Fig. 2).

Fig. 4 shows the spectrum of the mechanical signal measured using this scheme. The majority of the peaks shown are lip flapping, radial and rocking modes of the GOBLiT. The modes of interest (labeled 1 and 2) are the $n=2$ and $n=3$ wineglass modes of the GOBLiT. This measurement was carried out in air, with the quality factor of the $n=2$ wineglass mode calculated to be 206 . The wineglass modes were identified with a spectral scan of the resonance frequencies of the GOBLiT using a Laser Doppler Vibrometer (LDV) setup followed by 2D surface scan by raster scanning the LDV laser over the entire device to map out each mode. This is used to confirm the actual mode shape and enables the characterization of the spectrum for mode identification. Fig. 5 shows the spectral scan of the LDV with 2D maps of the modes of interest as insets.

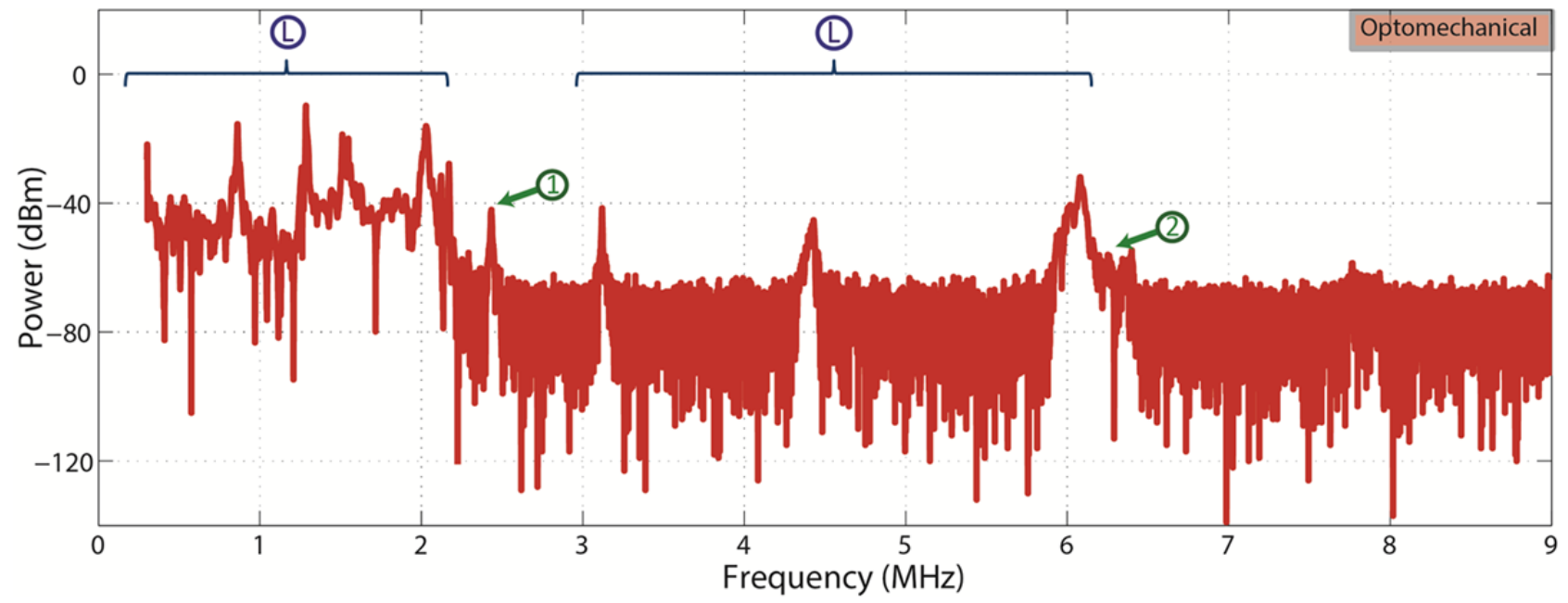

Figure 4: Mechanical frequency response measured using a fiber taper. Modes of interest are labeled 1 and 2 for the $n=2$ and $n=3$ wineglass resonance modes, respectively. Resonance modes marked with L refer to modes attributed to the lip or bowl extensional/tilting modes. 


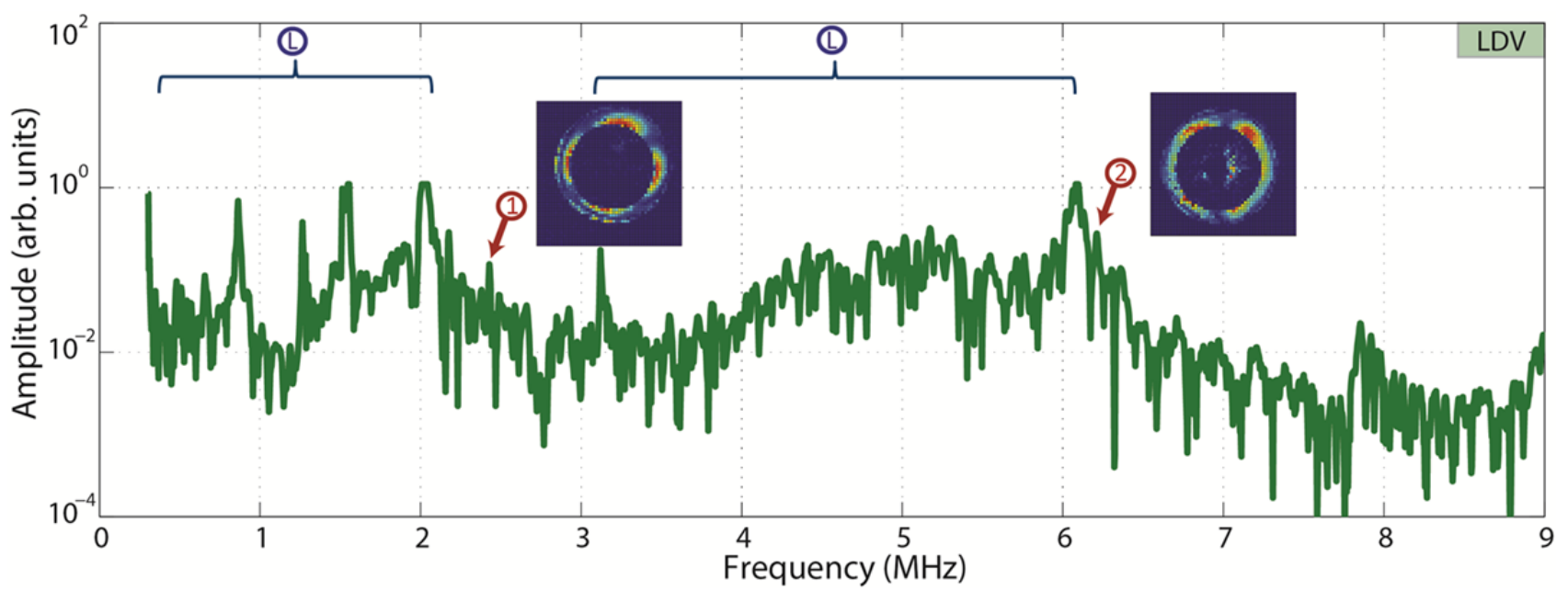

Figure 5: Mechanical response of GOBLiT measured using the LDV sweep. Modes labeled 1 and 2 are the $n=2$ and $n=3$ wineglass modes of the GOBLiT and the insets are the corresponding $2 D$ scan of the mode profile carried out using the LDV.

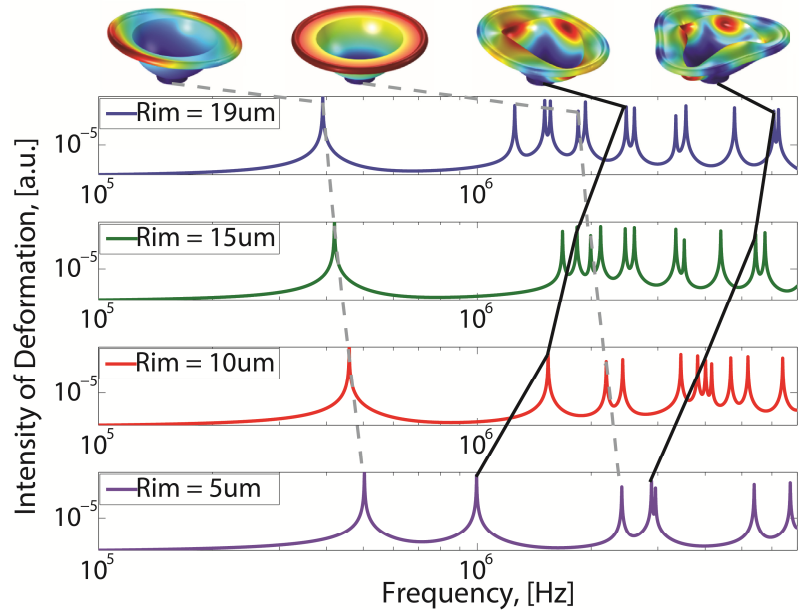

Figure 6: COMSOL simulation of mechanical modes of the GOBLiT with decreasing lip width. Solid lines track the resonance frequency of the $n=2$ and $n=3$ wineglass modes and it can be seen that they decrease as the lip width decreases. At the same time, the resonance frequencies of all the lip flapping modes increase, leaving behind the mode of interest. The device simulated matches the dimensions of the structure from Fig. $1 \mathrm{~g}$ with a radius of $60 \mu \mathrm{m}$ and oxide thickness of $1.1 \mu \mathrm{m}$.

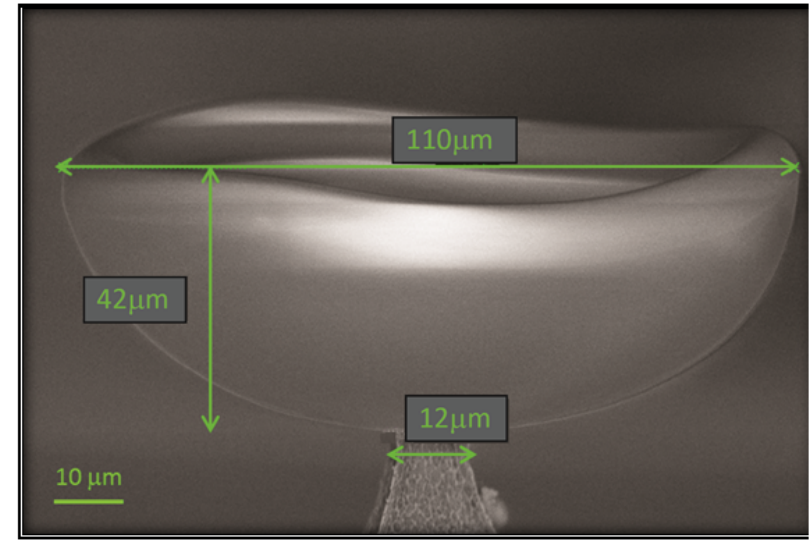

Figure 7: SEM of a GOBLiT with fully reflowed lip.

To understand the dependence of all the modes on the various dimensions of the device, we carried out simulations in COMSOL. As expected, the majority of modes are primarily dependent on the width of the lip, as shown in Fig. 6. As the width of the rim is reduced, the resonance frequencies of the parasitic modes increase and are separated from the $n=2$ wineglass mode.

To obtain smaller lip width of the GOBLiT, we modified the fabrication process. At the step of partial release in Fig. 1(d), we continue the $\mathrm{XeF}_{2}$ etch until the edge of the rim meets the bowl.

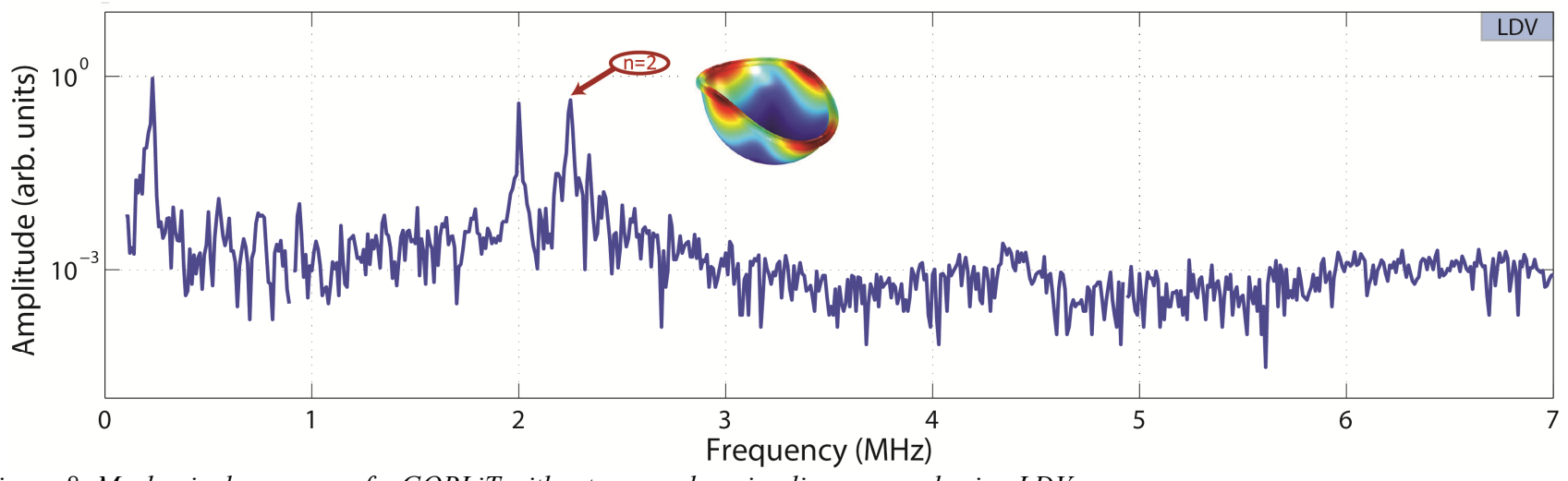

Figure 8: Mechanical response of a GOBLiT without an overhanging lip measured using LDV. 
This allows us to reflow the oxide further in towards the rim, moving the lip flapping modes to higher frequencies, or completely eliminating them. Fig. 7 shows an SEM image of such a GOBLiT showing the fully reflowed lip. Fig. 8 presents the measured LDV frequency response of the device showing a cleaner spectrum in the absence of lip flapping modes. However, we were not able to perform the analogous optomechanics measurement due to uneven reflow of the rim as seen in Fig. 7. This highlights the importance of the need for a symmetric undercut etch as the subsequent reflow step is critically dependent on the undercut.

\section{CONCLUSION}

We have demonstrated the first guided light measurement of the mechanical modes of a GOBLiT structure. By modifying the fabrication process we were able to incorporate a toroidal optical cavity on the GOBLiT for higher optical quality factor, as well as reduced parasitic mechanical modes of vibration in the region of our modes of interest. The effect of the width of the lip was simulated and experimentally measured using LDV. Continued improvement of the fabrication process will allow pure optical drive and sense of the mechanical modes, and make viable the use of this structure as a fully optomechanical sensor.

\section{ACKNOWLEDGEMENT}

The authors would like to thank DARPA MRIG program for sponsoring this research. The devices were fabricated in the Cornell Nanoscale Science and Technology Facility (CNF). Travel support has been generously provided by the Transducer Research Foundation.

\section{REFERENCES}

[1] A Schliesser, G Anetsberger, R Rivière, O Arcizet and T J Kippenberg, "High-sensitivity monitoring of micromechanical vibration using optical whispering gallery mode resonators", New J. Phys. 10095015 (2008).
[2] D. M. Rozelle "The hemispherical resonator gyro: From wineglass to the planets (AAS 09-176)", Proc. 19th AAS/AIAA Space Flight Mechanics Meeting, pp.1157 -1178 2009.

[3] L. Sorenson, P. Shao, and F. Ayazi, "Effect of thickness anisotropy on degenerate modes in oxide micro-hemispherical shell resonators" IEEE International Conference on Micro Electro Mechanical Systems (MEMS 2013), Taibei, Taiwan, Jan. 2013, pp. 169-172.

[4] M.L. Chan ,J. Xie, P. Fonda, H. Najar, K. Yamazaki, L. Lin and D.A. Horsley, "Micromachined Polycrystalline Diamond Hemispherical Shell Resonators" Hilton Head 2012 SolidState Sensors, Actuators \& Microsystems Workshop, Hilton Head Island, SC, Jun. 3-7, 2012.

[5] Doruk Senkal, Christopher R. Raum, Alexander A. Trusov, Andrei M. Shkel, "Titania Silicate / Fused Quartz Glassblowing for 3-D Fabrication of Low Internal Loss Wineglass Micro-structures”, Solid-State Sensors, Actuators, and Microsystems Workshop 2012, Hilton Head Island, South Carolina, USA, June 3-7, 2012.

[6] L. C. Fegely, D. N. Hutchison, S. A. Bhave, "Isotropic Etching of 111 SCS for Wafer Scale Manufacturing of Perfectly Hemispherical Silicon Molds", Transducers '11, pp. 2295-2298, Beijing, China, 2011.

[7] A. Heidari, M.-L. Chan, H.-A. Yang, G. Jaramillo, P. TaheriTehrani, P. Fonda, H. Najar, K. Yamazaki, L. Lin, D.A. Horsley, "Micromachined Polycrystalline Diamond Hemispherical Shell Resonators", Transducers '13, pp. 24152418, Barcelona, Spain, 2013.

[8] T.J. Kippenberg and K.J. Vahala, "Cavity Opto-Mechanics", Optics Express 15, 17172 (2007).

\section{CONTACT}

*Ajay K. Bhat, tel: +1-607-220-7146; akb96@cornell.edu 